\title{
Various ambiguities in re-constructing laser pulse parameters
}

\author{
Chandrasekhar Roychoudhuri ${ }^{\mathrm{a}}$ and Narasimha Prasad ${ }^{\mathrm{b}}$ \\ chandra@phys.uconn.edu \\ ${ }^{a}$ Physics Department, University of Connecticut, Storrs, CT 06269-5192 \\ ${ }^{\mathrm{b}}$ NASA Langley Research Center, 5 N. Dryden St., MS 468, Hampton, VA 23681
}

We think that mode lock laser pulses are generated by the summation process that take place between the monochromatic EM filed frequencies as if they interact with each other [1]:

$$
E\left(v_{0}, t\right)=\sum_{n=0}^{N-1} e^{-i 2 \pi\left(v_{0}+n \delta v\right) t+i \phi_{c}}=\frac{\sin N \pi \delta v t}{\sin \pi \delta v t} e^{-i 2 \pi v_{0} t+i \phi_{c}} \equiv a(t) e^{-i 2 \pi v_{0} t+i \phi_{c}}
$$

In reality, the pulse generation is a collaborative interaction process between EM fields and various material medium. When we carry out the actual mode lock analysis, we do take into account of interpaly between all the tempral dynamics of the cavity gain medium, cavity round trip time and the response time of the intra cavity element (saturable absorber, Kerr medium, etc.). that really enforces the "locking" of the phase of the cavity spontaneous emissions. On a conceptual level, this simplistic representation of the "mode locking" by Eq.1 ignores all these critical physical processes. When we try to analyze a pulsed field, again we start by representing it very much like this equation, even though we can only detect the square modulus of this complex field and loose a lot of phase related information to the detectors' quantum whims and their time constants [2].

The key parameters for a light pulse are as follows. Foremost is the (i) carrier frequency, which cannot be described or imagined without its state of undulation expressed as its (ii) phase. Next is our imagined time finite (iii) carrier envelope that provides the temporal boundary of the field amplitude strength of the undulating E-field. The final parameter is the (iv) state of polarization or the unique plane along which the strength of the E-field gradient undulates. None of these filed characteristics are made self-evident to us by the fields themselves. We do not see light. Light does not see light. Light beams pass through each other without altering each others energy distribution unless there are interacting material molecules (dipoles) within the physical volume of superposition of the beams. In contrast, we can sense the material particles. Material particles sense each other and they cannot pass through each other without interacting with (scattering from) each other. Thus the interpretation of the superposition phenomenon of multiple fields on detectors should not be lumped under the mysterious "wave-particle duality" philosophy. The phenomenon of superposition can be understood better when we focus on the actual process experienced by the detecting dipoles - when allowed by QM rules, they respond to and sum all the induced stimulations due to all the superposed fields followed by the proportionate energy absorption giving rise to the "fringes" we observe.

When the E-field gradient and its associated four parameters carry out a simple monotonic sinusoidal undulation, our life is very simple. Both conceptually and experimentally; classical optics is built upon such a premise. However, the real challenge is to characterize the random "temporal chirping" of all these four parameters during a very short period of time. This is simply an insurmountable problem as the signal duration approaches less than a single cycle. First, because the definition of frequency becomes meaningless, irrespective of how you want to define it. Second, we "see" EM fields only through the "eyes" of the various detectors wearing different "quantum goggles", none of which can respond to any signal instantaneously. Any material or dipole-like entity in this universe will need a finite amount of time to respond to any external stimulation because of their inertial mass; there cannot be any interaction that can be described as truly instantaneous. If the detecting dipole's response time is shorter than a cycle of the incident stimulating field, we have a high probability that it will be able to follow the stimulation induced by the carrier frequency (forced oscillation). If its response time is much longer than the total duration of the inducing field, it will appear as an "instantaneous impulse" to it. Since, the response time period of most atoms and molecules are in the fs or sub-fs domain, the initial dipole response optical fields are expressed as:

$$
\vec{p}(t)=\chi_{(1)} \vec{E}(t)+\chi_{(2)} \vec{E}^{2}(t)+\chi_{(3)} \vec{E}^{3}(t)+\ldots
$$

When the polarizable medium is crystalline, the representations for the vectors $\vec{p}(t), \chi_{(n)}$ and $\vec{E}^{n}(t)$ are quite complex [3]. The duration of the E-field and the other existing or controllably imposed local environmental fields around the responding dipoles will preferentially accentuate the specific n-th susceptibility $\chi_{(n)}$, or even a set of desired polarizing susceptibilities at the same time. The consequent report to us by a detector as to what it "sees" 
could indeed be quite varied and may even appear "schizophrenic" to us if we fail to understand the complexities of the time varying stimulations and the various time constants of the material medium. But many scientists have learnt to device a host of new atomic and molecular probing tools to study molecular dynamics by sending a sequence of controlled stimulating signals to extract new information out of the material dipoles. Others have devised new laser machines that achieve the desired perfections (like creating photonic crystals, etc.) by sending a train of laser impulses with varying time duration and intervals. These advances in technologies clearly indicate that we do understand quite a lot of the light-matter interaction processes even though we use the phrase "interference of light".

Unfortunately, we are still holding on to definitions for spectrum and coherence [4] that avoids any direct reference to the roles of the "reporters" (detecting dipoles and instrumental arrangements) who really provide us with any and all information that we ever can gather about the EM fields and the material universe. (Even our personal imaginations are generated by the neuro-chemical reactions.) It is true that the detectors are trustworthy and objective in their report because they are always self-consistent. But they are also always very much "subjective" because their behavior is always dictated by each one's unique quantum personal characteristics. Further, we do not know all of these quantum quirks of any single detecting entity in this universe, definitely no yet! Do we know what an electron really is!

Optical spectrum should be defined as the actual carrier frequency content generated by the source that produced the time finite undulations (carrier envelope) of the E-fields. The detecting molecules do not wait to respond to the signal to first evaluate the shape and duration of the particular carrier envelope, so they can respond to the appropriate set of Fourier frequencies of different phases and strengths. One of the consequences of this realization is that the corollary of the Fourier theorem, $(\delta v$-Fourier) $(\delta t$-carrier envelope $)>1$, is not a fundamental limit of nature, even though we tend to accept so due to various limitations of our detection systems that have nothing to do with the Fourier theorem. Atoms and molecules do not have long enough memories to learn Fourier theorem! So, you are free to invent new tools to achieve spectral super resolution of pulsed light [5].

Similarly, the autocorrelation (or the coherence) function should be defined in terms of the response of the detecting dipoles [6], whether we are using direct energy absorbing photo detectors or nonlinear EM field frequency converting media. It is the detectors that carry out the functions of first summing the effect of joint stimulations by all the superposed fields then absorb/convert the proportionate amount of energy as square modulus of the resultant complex stimulation (amplitude). EM fields do not carry out this processes as they do not interact with each other. Further, considering sub-fs response times of most molecules, EM fields are never functionally "incoherent" to each other. It is the integration times set by the detecting dipole and our detection systems that degrade the resultant fringe visibility (correlation function) that we measure (e.g., photographic plates to be exposed for development, finite electrical LCR response times to measure photo detector currents, etc.). We should not assign these measured degradations due to time averaging as the intrinsic "coherence" property of the fields.

Even though we can record only square modulus of the complex EM fields, the invention of holography provided us with the understanding that complex phase information can be registered as coded intensity variations through the cross product terms generated by the square modulus of the sum of the superposed fields on the detectors. Fortunately, the invention of the method of non-collinear second harmonic generation to characterize short pulses has captured the same fundamental technique, with the added benefit of being able to ignore the effect of the energy contained in the un-superposed segments of the replicated pulse pair.

We will present various experimental results to illustrate our arguments. Our position is that such detectorbehavior driven interpretations rather than the generally implied field-field interaction driven explanations, will help us better understand the ultimate nature of light and hence invent better and newer devices and instruments.

\section{References:}

1. (i) S. L. Shapiro, Ultrashort light pulses, Springer Verlag (1977). (ii)A. Siegman, Lasers, University Science Books (1986). (iii) R. Trebino, Frequency resolved optical gating, Kluwer Academic (2000).

2. C. Roychoudhuri, Phys.Essays 19 (3), September 2006, to be published, "Locality of superposition principle is dictated by detection processes".

3. D. L. Mills, Nonlinear Optics - Basic Concepts, Springer (1998).

4. (i) M. Born and E. Wolf, Principles of Optics, $6^{\text {th }}$ Ed., Cambridge U. Press (1980). (ii) L. Mandel and E. Wolf, Optical Coherence and Quantum Optics, Cambridge U. Press (1995).

5. C. Roychoudhuri and M. Tayahi, Int. J. of Microwave and Optics Tech., July 2006; "Spectral super-resolution by understanding superposition principle \& detection processes"; manuscript ID\# IJMOT-2006-5-46: http://www.ijmot.com/papers/papermain.asp.

6. C. Roychoudhuri, Proc. SPIE LASE Conference, Vol. 6108, paper \#50 (2006); "Reality of superposition principle and autocorrelation function for short pulses".

CR acknowledges partial support from the Nippon Sheet Glass Corporation. 